\title{
Guest Editorial: Special Issue on Machine Learning for Signal Processing
}

\author{
Ioannis Pitas • Vince Calhoun • \\ Konstantinos Diamantaras
}

Published online: 18 December 2009

(C) 2009 Springer Science+Business Media, LLC. Manufactured in The United States

Machine Learning (ML) is a generic term referring to methods and algorithms that learn based on empirical observations. In recent years, the field has matured considerably in both methodology and real-world application domains. ML methods belong not only to the classical supervised, unsupervised, or reinforcement learning paradigms (often associated with neural networks) but also to an increasingly wide range of methodologies including kernel methods, support vector machines (SVMs), Bayesian learning, etc. Also ML has become particularly important for the solution of problems in signal processing. Machine learning for signal processing combines many ideas from adaptive signal/image processing, optimization theory, learning theory and models, and statistics in order to solve complex real-world signal processing applications. The range of applications is also growing, including pattern recognition,

\footnotetext{
I. Pitas $(\bowtie)$

Aristotle University of Thessaloniki,

Thessaloniki 54124, Greece

e-mail: pitas@aiia.csd.auth.gr

V. Calhoun

The Mind Research Network,

Albuquerque, NM 87131, USA

e-mail: vcalhoun@unm.edu

K. Diamantaras

TEI of Thessaloniki,

Sindos 57400, Greece

e-mail: kdiamant@it.teithe.gr
}

adaptive filtering, computer vision, content based image and video retrieval, data mining, cognitive radio, robot control, data fusion, blind signal processing, sparse component analysis, brain-computer interfaces, etc.

This special issue has been put together from extended versions of papers presented in the 2007 IEEE International Workshop on Machine Learning for Signal Processing (MLSP-2007), held in Thessaloniki, Greece, between August 27 and 29, 2007. The guest editor committee invited the authors of the top ranking papers - based on the review scores they received at MLSP-2007-to submit their extended papers to the special issue. The committee also decided to invite the keynote and plenary speakers to submit their contributions in a full paper format. All papers went through a regular reviewing process and were duly revised, if necessary, prior to acceptance. Since the workshop covered the overall area of machine learning the papers represent a wide range of topics including feature-extraction and classification, nonlinear learning methods, speech separation, image and video processing, inference and programming methods.

We would like to take this opportunity to thank the rest of the organizing committee of the workshop for their help and support: Tülay Adal1, University of Maryland, Baltimore County, USA, Jan Larsen, Technical University of Denmark, Theophilos Papadimitriou, University of Thrace, Greece, Marc Van Hulle, Katholieke Universiteit Leuven, Belgium, Scott Douglas, Southern Methodist University, TX, USA, and Deniz Erdogmus, Oregon Health \& Science University, OR, USA. 


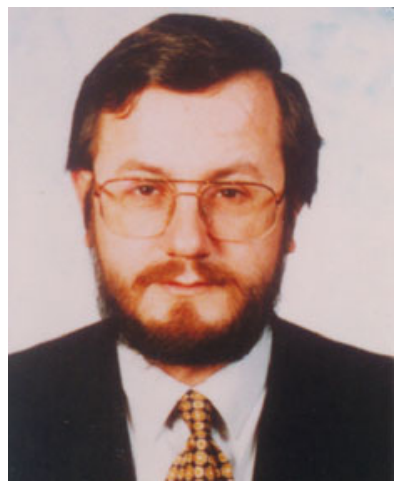

Ioannis Pitas received the Diploma of Electrical Engineering in 1980 and the $\mathrm{PhD}$ degree in Electrical Engineering in 1985 both from the Aristotle University of Thessaloniki, Greece. Since 1994, he has been a Professor at the Department of Informatics, Aristotle University of Thessaloniki. From 1980 to 1993 he served as Scientific Assistant, Lecturer, Assistant Professor, and Associate Professor in the Department of Electrical and Computer Engineering at the same University. He served as a Visiting Research Associate or Visiting Assistant Professor at several Universities. He has published over 640 papers and contributed in 32 books in his areas of interest and edited or coauthored another 7. He has also been an invited speaker and/or member of the program committee of several scientific conferences and workshops. In the past he served as Associate Editor or co-Editor of four international journals and General or Technical Chair of three international conferences. His current interests are in the areas of digital image and video processing and analysis, multidimensional signal processing, watermarking and computer vision.

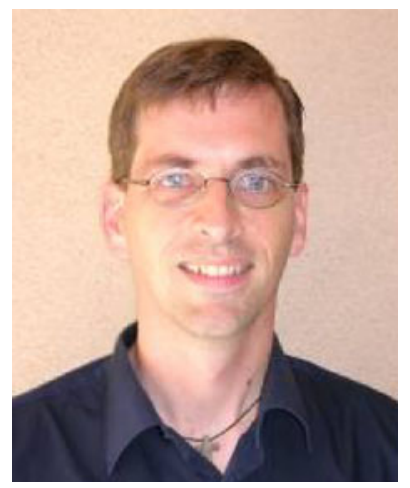

Vince Calhoun received a bachelor's degree in Electrical Engineering from the University of Kansas, Lawrence, Kansas, in 1991, master's degrees in Biomedical Engineering and Information Systems from Johns Hopkins University, Baltimore, in 1993 and 1996, respectively, and the Ph.D. degree in electrical engineering from the University of Maryland Baltimore County, Baltimore, in 2002. He worked as a Senior Research Engineer at the Psychiatric Neuro-Imaging Laboratory at Johns Hopkins from 1993 until 2002. Then he took a position as the director of medical image analysis at the Olin Neuropsychiatry Research Center and an associate professor at Yale University.

Dr. Calhoun is currently Chief Technology Officer and Director of Image Analysis and MR Research at the Mind Research Network and is an associate professor in the Departments of ECE, neurosciences, and computer science at the University of New Mexico. He is the author of more than 100 full journal articles, over 200 technical reports, abstracts and conference proceedings. Much of his career has been spent on the development of data driven approaches for the analysis of functional magnetic resonance imaging (fMRI) data. $\mathrm{He}$ has won over \$12 million in NSF and NIH grants on the incorporation of prior information into independent component analysis (ICA) for fMRI, data fusion of multimodal imaging and genetics data, and the identification of biomarkers for disease.

Dr. Calhoun is a senior member of the IEEE, the Organization for Human Brain Mapping, and the International Society for Magnetic Resonance in Medicine. He has participated in multiple NIH study sections. He has worked in the organization of workshops at conferences including the society of biological psychiatry (SOBP) and the international conference of independent component analysis and blind source separation (ICA). He is currently serving on the IEEE Machine Learning for Signal Processing (MLSP) Technical Committee and has previous served as the general chair of the 2005 meeting. He is a reviewer for a number of international journals and is on the editorial board of the Human Brain Mapping and Neuroimage journals and an associate editor for the IEEE Signal Processing Letters and the International Journal of Computational Intelligence and Neuroscience.

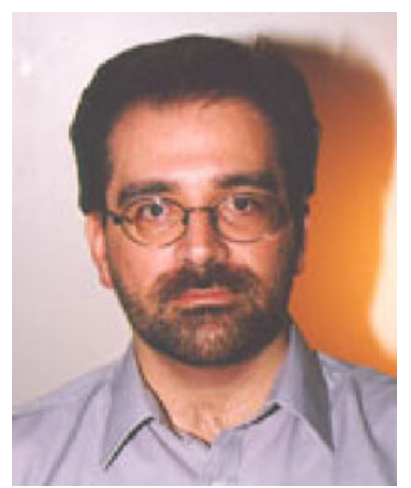

Konstantinos Diamantaras was born in Athens, Greece, in 1965. He received his Diploma in Electrical Engineering from the National Technical University of Athens in 1987 and the Ph.D. degree, also in electrical engineering, from Princeton University, Princeton, NJ, in 1992. Subsequently, he joined Siemens Corp. Research, Princeton, as a post-doctoral researcher, and in 1995, he worked as a researcher with the Department of Electrical and Computer Engineering, Aristotle University of Thessaloniki, Thessaloniki, Greece. Since 1998, he has been with the Department of Informatics, Technological Education Institute of Thessaloniki, where he currently holds the position of professor.

His research interests include signal processing, neural networks, image processing, and VLSI array processing. Since 1997, he has been serving as an editor for the Journal of VLSI for Signal Processing (Springer). Dr. Diamantaras is a Senior Member of IEEE and also serves as associate editor for the IEEE Transactions on Signal Processing and IEEE Signal Processing Letters. In 1997, he was a co-recipient of the IEEE Best Paper Award in the area of Neural Networks for Signal Processing. He is the author of the book Principal Component Neural Networks: Theory and Applications, co-authored with S.Y.Kung (New York: Wiley, 1996). He is currently chairman of the IEEE Machine Learning for Signal Processing (MLSP) Technical Committee and from 2005 to 2008 he was a member of the IEEE Signal Processing Theory and Methods (SPTM) TC. He has been the chairman of the 2007 IEEE Int. Workshop on Machine Learning for Signal Processing (MLSP2007) held in Thessaloniki, Greece, and also a technical committee member for various international signal processing and neural networks conferences. $\mathrm{He}$ is a member of the Technical Chamber of Greece. 